\title{
The Scalability and Performance of Common Vector Solution to Generalized Label Continuity Constraint in Hybrid Optical/Packet Networks
}

\author{
Shujia Gong and Bijan Jabbari \\ \{sgong, bjabbari\}@gmu.edu \\ George Mason University
}

\begin{abstract}
In hybrid Optical/packet networks, wavelength and VLAN tag continuity along a Label Switched Path are two common constraints. These two types of constraints, referred to as Generalized Label Continuity Constraints, have global significance in a network; namely, the label should appear only in other link- or node- disjoint paths. In considering these constraints, a solution is the common vector approach, which seeks a common available label along the path determined by CSPF without label constraints. In this paper, we provide an estimation method to compute the upper bound of the blocking probability for the above approach. We also address the scalability problem of the existing traffic engineering algorithms. We then present the simulation results corroborating with our analytical model.h.
\end{abstract}

\section{INTRODUCTION}

Traffic engineering utilizing Multi-Protocol Label Switching (MPLS) and Generalized MPLS (GMPLS)[1] are widely utilized in the backbone of the Internet infrastructure [2]. GMPLS is attractive as it allows end-to-end control, provisioning, protection and restoration in the hybrid IP and Optical transport networks. Nonetheless, there are challenging problems associated with the Label Switched Path (LSP) setup process due to physically diverse data forwarding plane with multiple routing and switching technologies. The path computation algorithms are needed to take into account of all forwarding technology and network specific constraints in order to yield an end-to-end LSP.

An example of the above constraints is the wavelength continuity constraint in All-Optical Networks (AONs) or networks based on Reconfigurable Optical Add-Drop Multiplexer (ROADM) and/or Dense Wavelength Division Multiplexing (DWDM), in which an end-to-end LSP needs to be setup. Deterministic network services to high-end eScience applications that require high speed and high capacity lightpath, which is subject to the wavelength continuity constraint, are in demand.

Another example is the VLAN tag continuity, where an endto-end path across geographically dispersed Ethernet switches is needed. This Ethernet switching technology is deployed not only in local area networks, but also in backbones such as in the hybrid optical and packet network infrastructure, e.g., HOPI project. Ethernet switches in the network may not necessarily support VLAN tag swapping and therefore, VLAN tag continuity constraint also needs to be satisfied.
Both wavelength and VLAN tags, as mentioned above, are labels. These as well as other labels can be classified under Generalized Label Continuity Constraints (GLCC). GMPLS defines a set of protocols as the standardized control plane to instantiate the LSP setup, tear-down and manipulation.

In this paper, we discuss the applicability of a common vector solution to address the GLCC problem. We develop an analytical model to obtain the estimation on blocking probability of LSP setup requests. We also develop a simulation model. Subsequently, numerical results are presented and compared with the analytical model. Finally, concluding remarks are made.

\section{COMMON VECTOR SOLUTIONS TO GLCC CONSTRAINTS}

An approach to solve the wavelength continuity constraint by converting a Network Graph to a Wavelength Graph has been presented in [3]. In wavelength graph, one plane is generated for each wavelength, and each node in the network graph is duplicated at each wavelength plane. For those nodes that have wavelength conversion capability, additional links are created to connect the replications of each node on corresponding wavelength plane. Virtual nodes are generated as a dummy originating node and a dummy destination node. These virtual nodes are connected to the replications of the true originating node and destination node respectively and metric on those virtual links is assigned as zero.

Although wavelength graph can find an optimal path for an LSP setup request, and can likewise be used to address VLAN continuity constraints, it has inherent scalability problems. The computational complexity of wavelength graph is $k n(n+k)$, where $k$ is the number of wavelength in a fiber and $n$ is the number of nodes in the network graph, which makes it impractical for large label space regardless of the network size.

\section{A. VLAN Tag Constraints and the Solution}

VLAN tag is a field of 12 bits, which can identify $k=4096$ VLANs. If we create VLAN plane as we do on wavelength plane and replicate nodes and links, a network will generate hundreds of millions of nodes and links. Clearly, wavelength graph approach cannot provide a scalable solution to VLAN tag continuity constraint.

To address this problem, we utilize the Common Vector solution, where the elements of the vector represent the availability or the lack of the labels at a node across the path. The set of available labels can simply be determined by taking 
the logical AND across the vectors at each node on the path. A method such as Extended Indexing Ref. [4] can be used to facilitate the distribution of the labels.

A vector comprised of 4096 bits, with each bit indicating the status of one of the 4096 VLAN tags used at each node is an attractive solution for large Ethernet networks in practice.

\section{B. Modeling of Common Vector Solution to Wavelength Continuity Constraint}

In this section, we show that the common vector approach is a reasonably good solution to wavelength continuity constraints by the following models.

We assume that the arrival process of LSP setup requests to each node is Poisson with mean of $\lambda$, and the departure process is exponential with mean service time $1 / \mu$

We use the following notations in our model:

E.

$G=<V, E>$ : Network graph $G$ with node set $V$ and link set

$N$ : Total number of nodes in $V$.

$M$ : Total number of links in $E$.

$L$ : Total number of labels in each link. In this context, labels are wavelengths.

$v_{i}$ : Node $i$ in node set $V$, where $i=1 \ldots N$.

$e_{i}$ : Link $i$ in link set $E$, where $i=1 \ldots M$.

$h_{i j}$ : The number of hops of the shortest path between $v_{i}$ and $v_{j}$ in $G$.

$\lambda_{i j}$ : The intensity of traffic with $v_{i}$ and $v_{j}$ as source and destination node, respectively.

$f_{i j}$ : The traffic flow generated by $\lambda_{i j}$ in the network.

$f_{e_{k}}$ : The total traffic flow on link $e_{k}$.

$I\left(v_{i}, v_{j}, e_{k}\right)$ : An indication function on whether the shortest path between $v_{i}$ and $v_{j}$ on the network graph traverses link $e_{k}$.

$A e_{i}$ : The number of available labels on link $e_{i}$.

$P_{i j}$ : Path between $v_{i}$ and $v_{j}$.

$p_{i j}^{m}$ : The link that the $m^{\text {th }}$ hop along $P_{i j}$ traverses.

$A_{p_{i j}^{1, \ldots, m}}$ : The number of labels that are commonly available on the first $m$ hops along $P_{i j}$.

We also assume that the traffic intensity in the network is not high. When an LSP request arrives at node $v i$, vi will check its Traffic Engineering Database (TEDB), prune those links without enough bandwidth and run CSPF without considering the label availability. After a path is found, vi will perform the logical AND operation on the vector of label availability along the path. If no commonly available label is found, the LSP request will be rejected.

To simplify the analysis, we assume that the label occupancy on each link is independent. This is not an accurate assumption in that all the labels along an LSP path are selected to be the same, which indicates the dependency of the label occupancy on different links. However, this dependency favors the common vector solution, and the actual performance of the network will be better than the analytical model we provide in the next subsections.

\section{Estimation of the Number of Available Labels on a Link}

Blocking probability in various network scenarios have been extensively studies, such as in [5]-[7]. Computing the number of available labels on a link is a $M / M / m / m$ queueing problem. We first need to know the traffic flow on each link.

The traffic flow generated by any pair of nodes $v_{i}$ and $v_{j}$ is:

$$
f_{i j}=\lambda_{i j} h_{i j}
$$

We first run the shortest path first algorithm between each pair of nodes on a network graph without traffic and define:

$$
I\left(v_{i}, v_{j}, e_{k}\right)= \begin{cases}1 & \text { if } P_{i j} \text { traverses } e_{k} ; \\ 0 & \text { Otherwise. }\end{cases}
$$

Due to the assumption that the traffic intensity is not high, only a small proportion of LSPs will take a path other than the shortest one, and we can neglect the difference between the offered load and the carried load on a link. Therefore, $f e_{k}$ can be estimated as:

$$
f_{e_{k}}=\sum_{i=1}^{N} \sum_{j=1}^{N} f_{i j} I\left(v_{i}, v_{j}, e_{k}\right)
$$

With the assumption of light traffic intensity, we can also approximate the arrival of LSP requests to a link to be a Poisson process. Therefore, we can apply $M / M / m / m$ formula to find the number of available labels.

$$
P\left(A_{e_{i}}=k\right)=\frac{f_{e_{i}}^{k} / k !}{\sum_{j=0}^{L}\left(f_{e_{i}}^{j} / j !\right)}
$$

This estimation in Equation (4) is very rough especially when traffic intensity is moderate. It assumes static routing and overestimates the traffic flow on each link. Reference [8] provides a more accurate estimation on the upper bounds of link blocking probability in homogeneous optical ring networks.

If we assume that the routing algorithm is dynamic, the link blocking probability is nearly the same in the whole network, and the network is complicated enough that the alternative path is of the equal length as the shortest path, we can further simplified the equation (3) as follows:

$$
f_{e_{k}}=\frac{\sum_{i=1}^{N} \sum_{j=1}^{N} f_{i j}}{M}
$$

Because we use offered load instead of carried load to calculate the traffic flow on each link, the equation (4) definitely gives us the upper bound of the actual blocking ratio.

\section{Estimation of Blocking Probability of LSP Setup Requests}

Equation (4) gives the probability of $P\left(\mathrm{Ae}_{\mathrm{i}}=k\right)$. For an LSP with multi-hops, a successful connection needs at least one particular label that is commonly available along the path.

Given a two-hop LSP $P_{i j}$ between $v_{i}$ and $v_{j}$, we denote that there are $k$ common wavelength available on $p_{i j}^{1}$ and $p_{i j}^{2}$ as $P\left(A_{p_{i j}^{1,2}}=k\right)$. 
Given $A_{p_{i j}^{1}}=k_{1}$ and $A_{p_{i j}^{2}}=k_{2}$, we have:

$$
\begin{array}{r}
P\left(A_{P_{i j}^{1,2}}=k\right)=\sum_{k_{1}=0}^{L} \sum_{k_{2}=0}^{L}\left(P \left(A_{P_{i j}^{1,2}}=k \mid A_{P_{i j}^{1}}=k_{1},\right.\right. \\
\left.\left.A_{P_{i j}^{2}}=k_{2}\right) P\left(A_{P_{i j}^{1}}=k_{1}\right) P\left(A_{P_{i j}^{2}}=k_{2}\right)\right)
\end{array}
$$

Given $L$ labels in a link, the number of possibilities for $A_{p_{i j}^{1}}=k_{1}$ and $A_{p_{i j}^{2}}=k_{2}$ is:

$$
\left(\begin{array}{l}
L \\
k_{1}
\end{array}\right)\left(\begin{array}{l}
L \\
k_{2}
\end{array}\right)
$$

Given $L$ labels in a link, $A_{p_{i j}^{1}}=k_{1}$ and $A_{p_{i j}^{2}}=k_{2}$, $A_{p_{i j}^{1,2}}=k$ means that we choose $k_{1}$ out of $L$ on $p_{i j}^{1}$ first. Because there are $k$ common labels on $p_{i j}^{1}$ and $p_{i j}^{2}$, it means that we choose $k$ labels out of $k_{1}$ labels on $p_{i j}^{1}$ and $k_{2}-k$ labels out of $L-k_{1}$ labels on $p_{i j}^{2}$.

The number of possibilities is:

$$
\left(\begin{array}{l}
L \\
k_{1}
\end{array}\right)\left(\begin{array}{l}
k_{1} \\
k
\end{array}\right)\left(\begin{array}{l}
L-k_{1} \\
k_{2}-k
\end{array}\right) .
$$

Dividing (8) by (7), we have:

$$
P\left(A_{P_{i j}^{1,2}}=k \mid A_{P_{i j}^{1}}=k_{1}, A_{P_{i j}^{2}}=k_{2}\right)=\frac{\left(\begin{array}{l}
k_{1} \\
k
\end{array}\right)\left(\begin{array}{l}
L-k_{1} \\
k_{2}-k
\end{array}\right)}{\left(\begin{array}{l}
L \\
k_{2}
\end{array}\right)},
$$

where $\max \left(0, k_{1}+k_{2}-L\right) \leq k \leq \min \left(k_{1}, k_{2}\right)$.

Therefore, we have:

$$
\begin{aligned}
& P\left(A_{P_{i j}^{1,2}}=k\right)= \\
& \sum_{k_{1}=1}^{L} \sum_{k_{2}=1}^{L} \frac{\left(\begin{array}{l}
k_{1} \\
k
\end{array}\right)\left(\begin{array}{l}
L-k_{1} \\
k_{2}-k
\end{array}\right)}{\left(\begin{array}{l}
L \\
k_{2}
\end{array}\right)} P\left(A_{P_{i j}^{1}}=k_{1}, A_{P_{i j}^{2}}=k_{2}\right)
\end{aligned}
$$

where $\max \left(0, k_{1}+k_{2}-L\right) \leq k \leq \min \left(k_{1}, k_{2}\right)$.

The estimation of the case that there are $k$ common labels along a $n$-hop LSP is an iterative process. We have:

$$
\begin{array}{r}
P\left(A_{P_{i j}^{1,2, \ldots, n}}=k\right)=\sum_{k_{1}=0}^{L} \sum_{k_{2}=0}^{L}\left(P \left(A_{P_{i j}^{1,2, \ldots, n}}=k \mid A_{P_{i j}^{n}}=k_{2},\right.\right. \\
\left.\left.A_{P_{i j}^{1, \ldots, n-1}}=k_{1}\right) P\left(A_{P_{i j} \ldots, n-1}=k_{1}\right) P\left(A_{P_{i j}^{n}}=k_{2}\right)\right)
\end{array}
$$

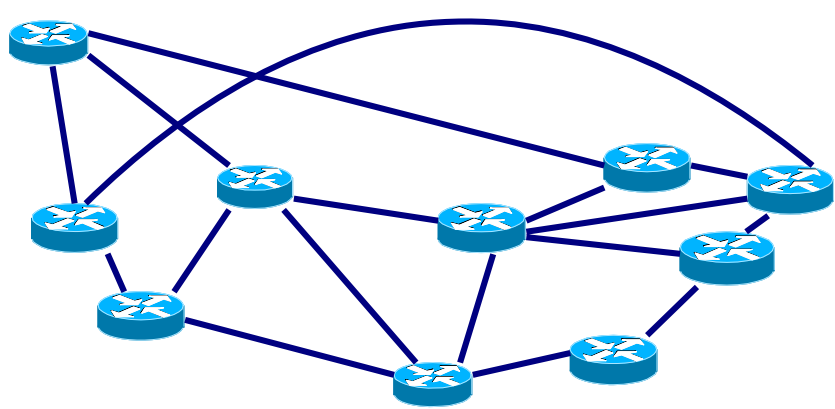

Fig. 1. Network Topology

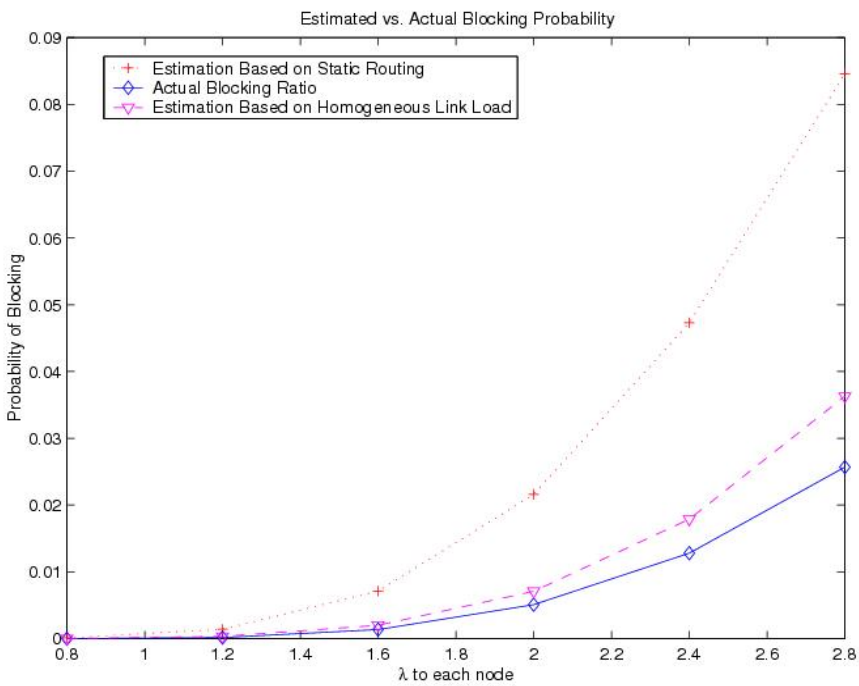

Fig. 2. Comparison of Estimated and Actual Blocking

Therefore, the probability of blocking is $P\left(A_{P_{i j}^{1,2, \ldots, n}}=0\right)$.

We assume that the alternative path between any pair of nodes $v_{i}$ and $v_{j}$ is of the same length as the shortest path. The expected blocking probability is as follows:

$$
P_{b}=\frac{\sum_{i=1}^{N} \sum_{j=1}^{N} \lambda_{i j} P\left(A_{P_{i j}^{1,2, \ldots, h_{j}}}=0\right)}{\sum_{i=1}^{N} \sum_{j=1}^{N} \lambda_{i j}}
$$

If we use (3) to estimate the traffic flow on a link, we called it estimation based on static routing. If we use (5) to estimate the traffic flow on a link, we called it estimation based on homogeneous link load assumption.

\section{SIMULATION VERSUS ANALYTICAL RESULTS}

We consider a network topology as depicted in Fig. 1, and assume that the traffic matrix is homogeneous. We consider that each link has 8 wavelengths.

Fig. 2 shows the estimation of blocking probability based on both static route and the homogeneous link load assumption respectively. It also showed the actual blocking probability by simulating 10 million LSP setup requests. We can clearly see 


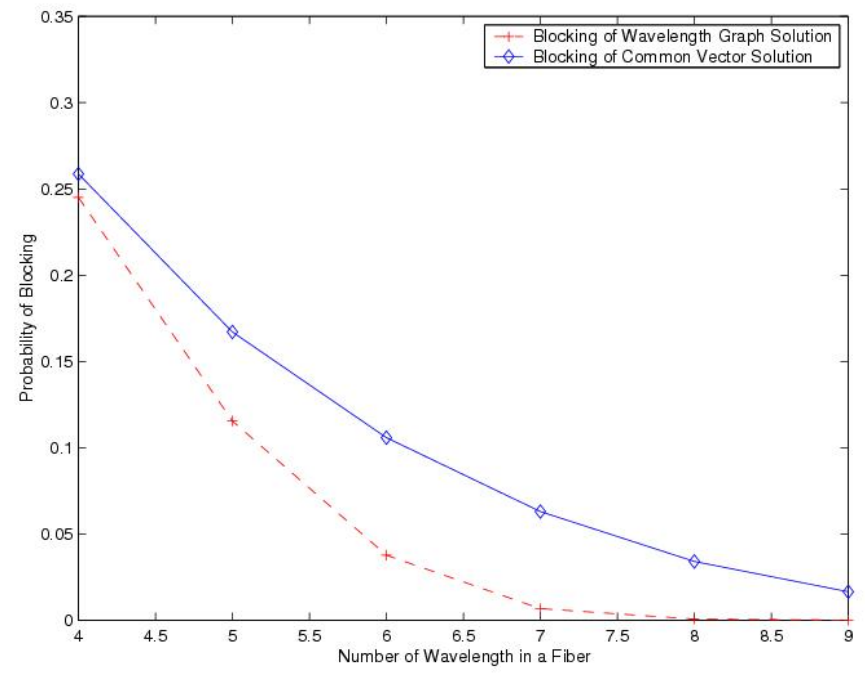

Fig. 3. Comparison of Actual Blocking in Wavelength Graph Solution and Common Vector Solution

that the estimation based on homogeneous link load assumption is closer to the actual blocking rate. Both estimations are upper bounds of the actual blocking rate.

Figure 3 also compared the blocking ratio of the common vector solution with the wavelength graph solution. Given incoming traffic to each node of 3 Erlangs, we simulated the cases that there are 4 to 8 wavelengths in a fiber. None of the node has wavelength translation capability. The wavelength graph solution can accept more calls than the common vector solution. However, when the network load is very high or very low, the improvement on the call acceptance ratio is not significant. When the network load is moderate, wavelength graph solution can show its advantage with regard to call acceptance.

The computational complexity of the common vector solution is equivalent to run Dijkstra algorithm and then find the availability of labels on each link traversed by the LSP. Therefore, it is $O(M+N \log (N))+O(N+L)$. The computational complexity of the wavelength graph solution is given in [3], which is $O(L N(N+L))$.

Figure 4 shows that the computational complexity of common vector solution is almost irrelevant to the number of wavelengths on a link, while the wavelength graph solution shows that even if the wavelength number is small, it still takes much more time than the common vector solution does.

Homogeneous and non-homogeneous traffic matrix may result in different network blocking scenario. To make the comparison, we design three cases:

1) Traffic generated at each node is the same and the traffic matrix is homogenous;

2) Traffic generated at each node is the same and the traffic matrix is non-homogenous, i.e. the mean holding time between node $v_{i}$ and $v_{j}$ is in proportion to the value in $i^{\text {th }}$ row and $j^{\text {th }}$ column given in table I; and

3) Traffic generated at each node is in proportion to the values given in table II and the traffic matrix is nonhomogenous as given in table I.

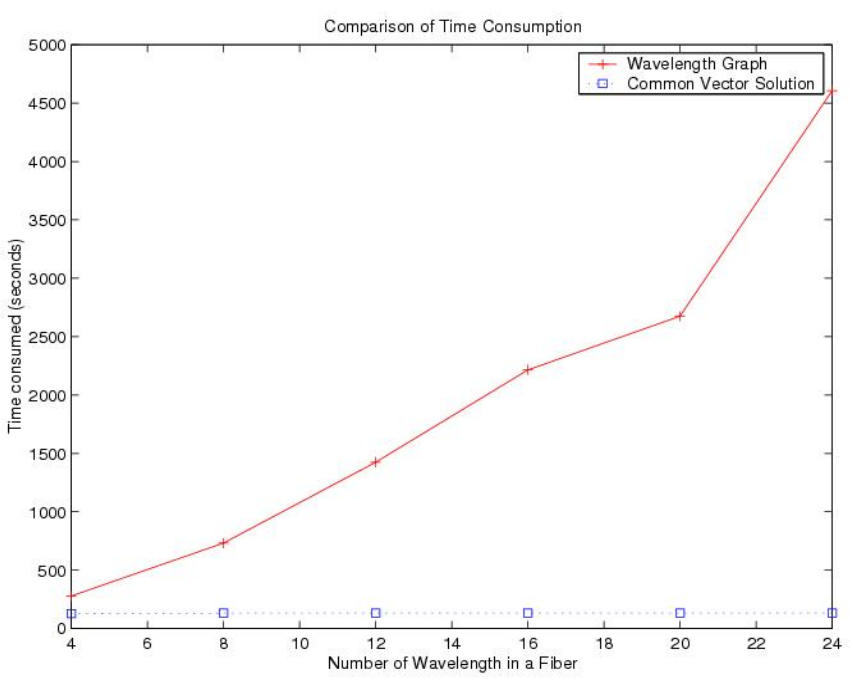

Fig. 4. Comparison of Time Consumption

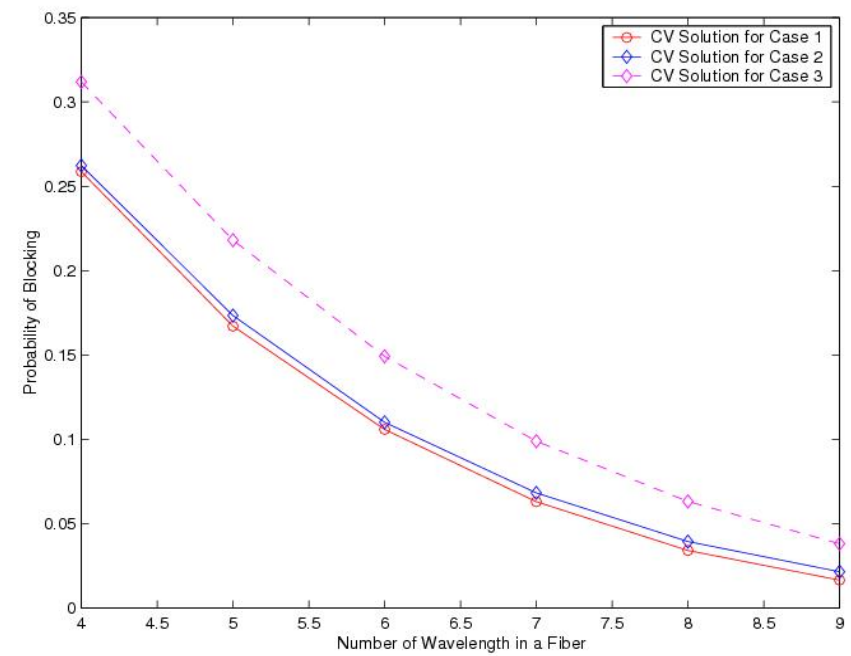

Fig. 5. Comparison of Blocking Probability of Common Vector Solution between Case 1, 2 and 3

TABLE I. NON-HOMOGENEOUS TRAFFIC MATRIX

\begin{tabular}{|c|c|c|c|c|c|c|c|c|c|c|}
\hline node & $\mathbf{0}$ & $\mathbf{1}$ & $\mathbf{2}$ & $\mathbf{3}$ & $\mathbf{4}$ & $\mathbf{5}$ & $\mathbf{6}$ & $\mathbf{7}$ & $\mathbf{8}$ & $\mathbf{9}$ \\
\hline $\mathbf{0}$ & 0.0 & 0.6 & 0.7 & 0.8 & 1.2 & 1.3 & 1.4 & 1.5 & 0.5 & 1.0 \\
\hline $\mathbf{1}$ & 0.6 & 0.0 & 0.7 & 0.9 & 0.8 & 1.0 & 1.1 & 1.2 & 1.4 & 1.3 \\
\hline $\mathbf{2}$ & 0.8 & 0.7 & 0.0 & 1.6 & 0.4 & 1.2 & 1.3 & 0.5 & 1.0 & 1.5 \\
\hline $\mathbf{3}$ & 0.8 & 0.7 & 1.6 & 0.0 & 1.2 & 0.4 & 1.2 & 0.8 & 1.3 & 1.0 \\
\hline $\mathbf{4}$ & 0.5 & 1.6 & 1.7 & 0.3 & 0.0 & 0.4 & 1.5 & 1.8 & 0.2 & 1.0 \\
\hline $\mathbf{5}$ & 1.8 & 0.8 & 0.2 & 1.2 & 0.9 & 0.0 & 1.1 & 1.8 & 0.2 & 1.0 \\
\hline $\mathbf{6}$ & 1.8 & 1.8 & 1.7 & 1.7 & 0.2 & 0.2 & 0.0 & 0.3 & 1.0 & 0.3 \\
\hline $\mathbf{7}$ & 1.8 & 1.5 & 1.1 & 0.5 & 0.9 & 0.5 & 0.2 & 0.0 & 1.5 & 1.0 \\
\hline $\mathbf{8}$ & 1.4 & 1.2 & 0.8 & 0.6 & 1.2 & 0.8 & 0.7 & 1.3 & 0.0 & 1.0 \\
\hline $\mathbf{9}$ & 1.8 & 1.8 & 1.7 & 0.3 & 1.0 & 0.2 & 0.8 & 0.2 & 1.2 & 0.0 \\
\hline
\end{tabular}

TABLE II. NON-HOMOGENEOUS TRAFFIC MATRIX WITH NONHOMOGENEOUS TRAFFIC INTENSITY AT EACH NODE

\begin{tabular}{|c|c|c|c|c|c|c|c|c|c|c|}
\hline node & $\mathbf{0}$ & $\mathbf{1}$ & $\mathbf{2}$ & $\mathbf{3}$ & $\mathbf{4}$ & $\mathbf{5}$ & $\mathbf{6}$ & $\mathbf{7}$ & $\mathbf{8}$ & $\mathbf{9}$ \\
\hline $\begin{array}{c}\text { Traffic } \\
\text { Intensity }\end{array}$ & 1.0 & 1.4 & 0.6 & 0.8 & 1.2 & 1.3 & 0.7 & 1.5 & 0.5 & 1.0 \\
\hline
\end{tabular}




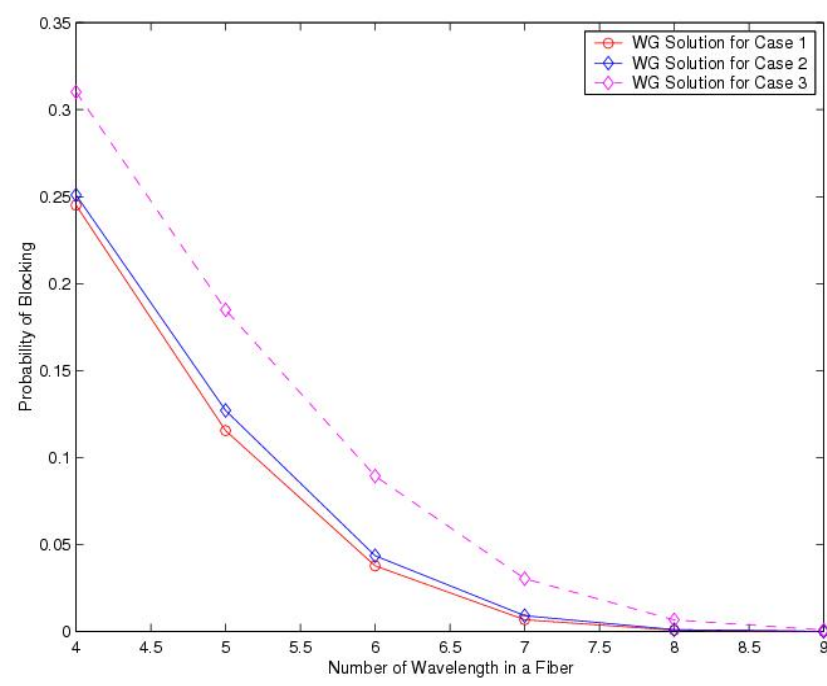

Fig. 6. Comparison of Blocking Probability of Wavelength Graph Solution between Case 1, 2 and 3

Figure 5 and 6 show the blocking probability of all the three cases of the common vector and wavelength graph solutions respectively. Comparing case 1 and case 2, there is not much performance degradation. However, case 3 is clearly worse than case 1 and case 2 . This implies that given the same traffic intensity, a network with nearly homogeneous traffic demand has better performance from blocking probability perspective.

Figure 7 shows the comparison between estimated and actual blocking probability of the common vector solution. The estimations based on both equation (3) and (5) are showed. When the traffic load is light, the estimation of blocking ratio based on dynamic routing is even less than the actual blocking ratio. This is because the dynamic routing assumption hides the non-homogeneous traffic demand from each node.

\section{CONCLUSION}

In this paper, we propose a scalable solution to the generalized label continuity constraints in an optical/packet hybrid network. We demonstrate that we can estimate the upper bound of the link blocking probability and show that this scalable solution will only slightly degrade the Quality of Service from the call blocking perspective, especially when the traffic load is light or the network is overloaded. This algorithm has been implemented in the DRAGON (Dynamic Resource Allocation in GMPLS Optical Networks) project, an NSF sponsored research to create dynamic, deterministic, and

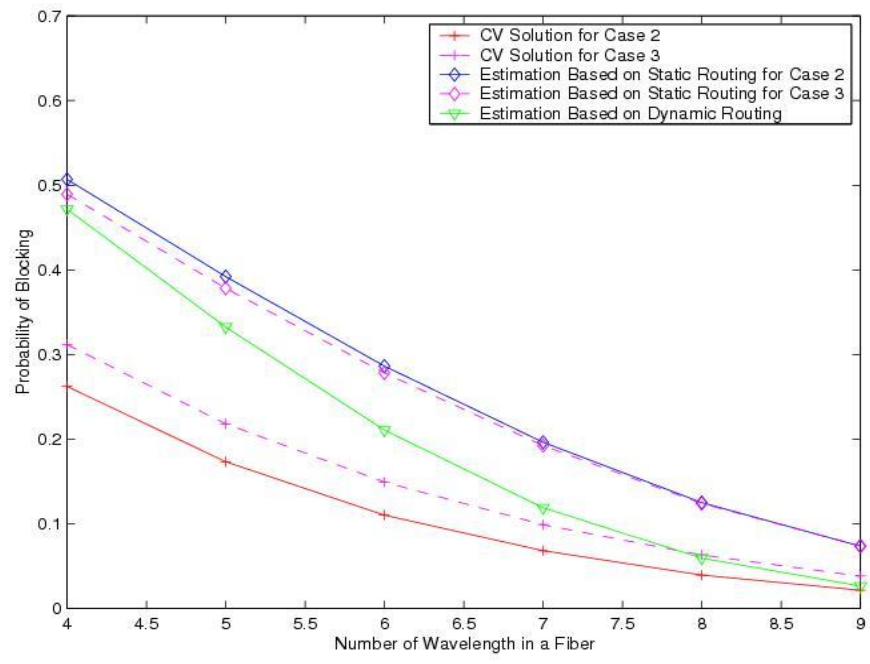

Fig. 7. Estimated vs. Actual Blocking Probability of Common Vector Solution in Case 1, 2 and 3

manageable end-to-end network transport services for high-end e-Science applications.

\section{REFERENCES}

[1] E. Mannie and Ed., "Generalized multi-protocol label switching (GMPLS) architecture,” RFC3954

[2] D. Awduche and B. Jabbari, "Internet traffic engineering using MultiProtocol Label Switching (MPLS),” Journal of Computer Networks, vol. 40, no. 1, pp. 111-129, Sept. 2002, invited Paper.

[3] I. Chlamtac, A. Faragoe, and T. Zhang, "Lightpath (Wavelength) routing in large WDM networks,” IEEE JSAC, vol. 14, no. 5, June 1996.

[4] B. Jabbari, "Extended indexing method for resource assignment," Internal Report, Aug. 2006.

[5] R. A. Barry and P. A. Humblet, "Models of blocking probability in alloptical networks with and without wavelength changers," IEEE Journal on Selected Areas in Communications, vol. 14, no. 5, pp. 858-867, June 1996.

[6] L. W. Chen and E. Modiano, "Efficient routing and wavelength assignment for reconfigurable wdm ring networks with wavelength converters,” IEEE/ACM Transactions on Networking, vol. 13, no. 1, pp. 173-186, Feb.2005.

[7] S. Subramaniam, M. Azizogelu, and A. K. Somani, "All-optical networks with sparse wavelength conversion," IEEE/ACM Transactions on Networking, vol. 4, no. 4, pp. 544-557, Aug. 1996.

[8] S. Gong and B. Jabbari, "Link performance bounds in homogeneous optically switched ring networks," IEEE GLOBECOM, San Francisco, Nov. 2006. 